\title{
Angular distributions and BSM fits for rare B decays
}

\section{Sébastien Descotes-Genon*,}

Laboratoire de Physique Théorique (UMR8627),

CNRS, Univ. Paris-Sud, Université Paris-Saclay, 91405 Orsay, France

and IJCLab (UMR9012), CNRS, Université Paris-Saclay, 91405 Orsay, France

E-mail: descotes@th.u-psud.fr

I review the current status of the $B$ anomalies in $b \rightarrow$ sll transitions. After recalling the experimental situation, I discuss the elements of the Standard Model (SM) predictions and the patterns of New Physics arising in model-independent analyses, before discussing some of the models beyond the SM proposed to explain these patterns. I discuss possible ways of improving of our understanding of the situation using new modes and new observables.

18th International Conference on B-Physics at Frontier Machines - Beauty2019-

29 September $/ 4$ October, 2019

Ljubljana, Slovenia

${ }^{*}$ Speaker. 


\section{Introduction}

Due to the structure of the Standard Model (SM), some quark flavour transitions are expected to be particularly sensitive to intermediate states with high energy scales and possibly involving New Physics (NP) degrees of freedom. The presence of widely different scales (non-perturbative QCD, heavy-quark masses, electroweak interaction, NP) makes the computation of such processes a tough theoretical challenge. It can be tackled using a ladder of effective field theories to separate the different dynamical scales involved: most can be treated perturbatively, leaving only non-perturbative inputs involving long-distance QCD hadronisation effects.

Recent measurements from the LHCb, Babar and Belle experiments suggest several interesting deviations from the SM in $b$-quark decays. The first set of deviations occurs in $b \rightarrow c \ell v_{\ell}$, by comparing branching ratios involving $\tau$ and lighter leptons (although recent results from Belle seem to weaken this signal). The second set happens for $b \rightarrow s \ell \ell$, in muon observables (branching ratios, angular observables) and in ratios of branching ratios for electron and muon modes. The latter decay is suppressed in the SM and is expected to show sensitivity to NP effects, whereas the former is a tree-level transition in the SM and is less expected to show large deviations due to higher-energy phenomena. Interestingly, both channels suggest NP effects violating lepton-flavour universality, an unambiguous sign of NP [1].

In the following, I will focus on the deviations in $b \rightarrow s \ell \ell$ transitions. Their description requires a careful assessment of the theoretical uncertainties of the SM predictions, and these deviations can be studied either in model-dependent or model-independent approaches to NP. I will then discuss potential ways of improving our understanding of these modes, by considering new observables and decays not yet explored in detail.

\section{BSM fits for $b \rightarrow s \ell \ell$ transitions}

\subsection{Experimental situation and effective Hamiltonian approach}

The transitions $b \rightarrow s \ell^{+} \ell^{-}$are studied in detail in the LHCb, CMS and ATLAS experiments, as well as in the Belle and Belle II collaborations. Recent experimental results have shown interesting deviations from the SM [1]. Branching ratios for $B \rightarrow K\left(^{*}\right) \mu \mu, B_{s} \rightarrow \phi \mu \mu$ are found consistently lower than the SM expectations over the whole kinematic range, whereas deviations were observed for some of the $B \rightarrow K^{*} \mu^{+} \mu^{-}$angular asymmetries built specifically to yield clean theoretical predictions, in particular for the angular observable $P_{5}^{\prime}$ [2] at large $K^{*}$ recoil. The ratios

$$
R_{K(*)}=\frac{B r\left(B \rightarrow K\left(^{*}\right) \mu^{+} \mu^{-}\right)}{B r\left(B \rightarrow K\left({ }^{*}\right) e^{+} e^{-}\right)}
$$

were measured by $\mathrm{LHCb}$ showing deviations from SM expectations between 2.3 and $2.5 \sigma$, whereas Belle measured recently the same observables with larger uncertainties, leading to results in agreement with both the SM and previous LHCb measurements. These deviations from lepton flavour universality seem related to anomalies in $b \rightarrow s \mu \mu$ transitions, since there are no indications of deviations from the SM in the Belle and LHCb measurements for $b \rightarrow$ see branching ratios and angular observables (although these measurements are challenging and thus with larger uncertainties than for $b \rightarrow s \mu \mu$ ). Other observables, probing different aspects of the $b \rightarrow s \mu \mu$ transition, 

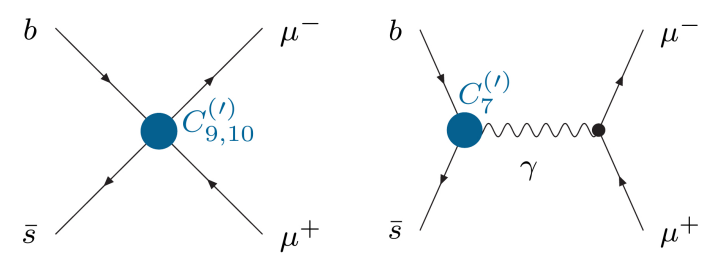

\begin{tabular}{c|c|c|c} 
processes & $\mathscr{C}_{7\left(^{\prime}\right)}$ & $\mathscr{C}_{9\left(^{\prime}\right) \mu}$ & $\mathscr{C}_{10\left({ }^{\prime}\right) \mu}$ \\
\hline \hline$B \rightarrow X_{s} \gamma, B \rightarrow K^{*} \gamma$ & $\mathrm{x}$ & & \\
\hline$B \rightarrow X_{s} \mu^{+} \mu^{-}$ & $\mathrm{x}$ & $\mathrm{x}$ & $\mathrm{x}$ \\
\hline$B_{s} \rightarrow \mu^{+} \mu^{-}$ & & & $\mathrm{x}$ \\
\hline$B_{(s)} \rightarrow\left(K^{(*)}, \phi\right) \mu^{+} \mu^{-}$ & $\mathrm{x}$ & $\mathrm{x}$ & $\mathrm{x}$ \\
\hline
\end{tabular}

Figure 1: Effective couplings $\mathscr{C}_{7\left({ }^{\prime}\right), 9\left(^{\prime}\right) \mu, 10\left(^{\prime}\right) \mu}$ contributing to $b \rightarrow s \mu \mu$ transitions and sensitivity of the various radiative and (semi)leptonic $B_{(s)}$ decays to them.

are in fair agreement with SM expectations, for instance the branching ratios for $B_{s} \rightarrow \mu \mu$ or the inclusive $B \rightarrow X_{s} \gamma$ decay.

The appearance of several tensions in different $b \rightarrow s \mu \mu$ channels is interesting since all the channels are dominantly sensitive to the same couplings $\mathscr{C}_{7\left(^{\prime}\right), 9\left(^{\prime}\right) \ell, 10\left(^{\prime}\right) \ell}$ induced by the operators in the effective Hamiltonian approach (see Fig. 1)

$$
\begin{array}{lll}
\mathscr{O}_{9\left(^{\prime}\right) \ell}=\frac{\alpha}{4 \pi}\left[\bar{s} \gamma^{\mu} P_{L(R)} b\right]\left[\bar{\mu} \gamma_{\mu} \mu\right], & \mathscr{O}_{10\left({ }^{\prime}\right) \ell}=\frac{\alpha}{4 \pi}\left[\bar{s} \gamma^{\mu} P_{L(R)} b\right]\left[\bar{\mu} \gamma_{\mu} \gamma_{5} \mu\right], & \mathscr{O}_{7\left(^{\prime}\right)}=\frac{\alpha}{4 \pi} m_{b}\left[\bar{s} \sigma_{\mu v} P_{R(L)} b\right] F^{\mu v}, \\
\mathscr{C}_{9 \ell}^{\mathrm{SM}}\left(\mu_{b}\right)=4.07, & \mathscr{C}_{10 \ell}^{\mathrm{SM}}\left(\mu_{b}\right)=-4.31, & \mathscr{C}_{7}^{\mathrm{SM}}\left(\mu_{b}\right)=-0.29,
\end{array}
$$

where $m_{b}$ denotes the $b$ quark mass and $\mu_{b}=4.8 \mathrm{GeV}$. Primed operators have vanishing or negligible Wilson coefficients in the SM, whereas the SM operators with the same structure but different lepton flavours have identical Wilson coefficients in the SM due to lepton-flavour universality. It is natural to ask whether a NP contribution to these couplings could account for all the tensions at once, coming from particular extensions of the SM inducing contributions to some Wilson coefficients. Indeed, the couplings $\mathscr{C}_{7,9,10}^{\left({ }^{\prime}\right)}$ can be constrained through various observables in radiative and (semi) leptonic $B_{(s)}$ decays, each of them sensitive to a different subset of coefficients (see Fig. 1).

\subsection{Hadronic uncertainties}

At leading order (LO) in the effective theory, SM predictions involve tree-level diagrams with insertions of the operators $\mathscr{O}_{7,9,10}$ (generated at one loop in the SM), as well as one-loop diagrams with an insertion of the charged-current operator $\mathscr{O}_{2}=\left[\bar{s} \gamma^{\mu} P_{L} c\right]\left[\bar{c} \gamma_{\mu} P_{L} b\right]$ (generated at tree level in the SM). In the first case the leptonic and the hadronic currents factorise, QCD corrections are restrained to the hadronic $B \rightarrow M$ current (first two diagrams in Fig. 2), leading to hadronic form factors parametrising the $B \rightarrow M$ transition. Contributions of the second type exhibit a non-local structure related to charm loops (third diagram in Fig. 2) that cannot be absorbed into form factors and that can become large when the $c \bar{c}$ pair becomes resonant (i.e. when the dilepton invariant mass becomes close to one of the charmonium resonances).

A first source of theoretical uncertainties comes from form factors, which are available from Lattice QCD (LQCD) as well as from light-cone sum rule (LCSR) calculations, with the former being suited for the region of high $q^{2}>15 \mathrm{GeV}^{2}$ (low $K^{*}$ recoil) and the latter for the region of low $q^{2}<8 \mathrm{GeV}^{2}$ (large $K^{*}$ recoil). Several determinations from light-cone sum rules have been proposed, using different formulations $[3,4]$. Some of these determinations have also been combined with LQCD results to provide a parametrisation over the whole kinematic range, with 

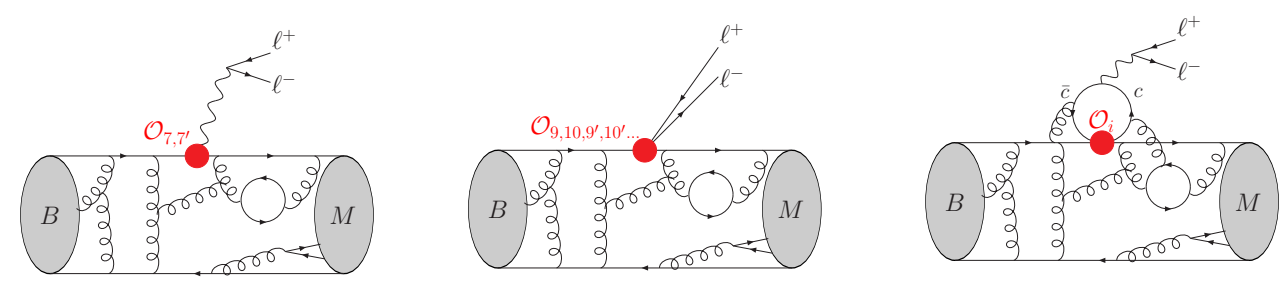

Figure 2: Illustration of local (first two diagrams) and non-local (third diagram) contributions to exclusive $B \rightarrow M \ell^{+} \ell^{-}$matrix elements [1].

very small error bars. However, these sum rules rely often on specific assumptions $\left(K^{*}\right.$ treated as an infinitely narrow resonance, contributions from higher excitations and/or higher-twist distribution amplitudes neglected) which should be investigated to attach a systematic uncertainty to these hypotheses [5]. For instance, it seems that treating the $K^{*}$ meson as a narrow resonance has some impact on the extraction of the form factors in the light-cone sum rules: the finite width of the $K^{*}$ could lead to a $10 \%$ enhancement of the form factors, increasing the discrepancy between SM expectations and measurements for $\operatorname{Br}\left(B \rightarrow K^{*} \mu \mu\right)$ (this effect is LFU and does not affect $R_{K^{*}}$ ).

Since the form factors introduce an important source of theoretical uncertainties and can be affected by signficant systematics, it is desirable to reduce the sensitivity of the predictions to these inputs as much as possible. For $B \rightarrow K^{*} \ell^{+} \ell^{-}$, this can be achieved in the low- $q^{2}$ region by exploiting large-recoil symmetries of QCD which relate the various hadronic form factors at LO in $\alpha_{s}$ and $\Lambda / m_{b}$ (where $\Lambda$ is the typical QCD scale). The coefficients of the differential angular distribution can then be used to build observables with a more limited sensitivity to the form factors by exploiting these relationships [6, 2,7], and the resulting optimised observables $P_{i}^{\left({ }^{\prime}\right)}$ at large recoil exhibit a mild form factor dependence, suppressed by powers of $\alpha_{s}$ and $\Lambda / m_{b}$.

A second source of uncertainties comes from $c \bar{c}$ loops. Long-distance charm-loop effects (third diagram in Fig. 2) can mimic an effective coupling $\mathscr{C}_{9}^{c \bar{c}}$. However, they have rather specific features, as their contribution is expected to have a non-constant $q^{2}$-dependence, where $q^{2}$ is the invariant mass of the lepton pair, and to be process dependent but LFU. Together with the perturbative SM contribution $\mathscr{C}_{9 \mathrm{SM} \text { ert }}^{\text {eff }}$ and a potential constant NP coupling $\mathscr{C}_{9}^{\mathrm{NP}}$, it can be cast into an effective Wilson coefficient $\mathscr{C}_{9}^{\text {eff i }}\left(q^{2}\right)=\mathscr{C}_{9 \mathrm{SM} \text { pert. }}^{\text {eff }}\left(q^{2}\right)+\mathscr{C}_{9}^{\mathrm{NP}}+\mathscr{C}_{9}^{c \bar{c} i}\left(q^{2}\right)$, with a different $\mathscr{C}_{9}^{c \bar{c} i}$ and hence also a different $\mathscr{C}_{9}^{\text {eff i }}$ for each transversity amplitude (for instance $i=0, \|, \perp$ for $B \rightarrow K^{*} \ell \ell$ ). The evaluation of this long-distance contribution is difficult, especially close to the region of charmonium resonances. Several estimates are available, based on light-cone sum rules, a sum of Breit-Wigner resonances or a general parametrisation relying on unitarity [3, 8, 9]. All agree well with each other and estimates for charm-loop effects are naturally included in the SM predictions for the various observables.

\subsection{Global analysis of $b \rightarrow s \ell \ell$ data}

The interest of a global analysis of various hadronic channels and observables was realised much before the advent of LHCb data [10]. The first analysis performed in this spirit and exploiting the LHCb 2013 data [11] pointed to a large negative contribution to the Wilson coefficient $\mathscr{C}_{9}$, as confirmed by other groups [12], and updated to take into account the recent measurements 


\begin{tabular}{crccc} 
Coefficient & Best fit & $1 \sigma$ & Pull & $p$-value \\
\hline $\mathscr{C}_{9}^{\mathrm{NP}}$ & -1.02 & {$[-1.18,-0.85]$} & 5.8 & $65 \%$ \\
$\mathscr{C}_{9}^{\mathrm{NP}}=-\mathscr{C}_{10}^{\mathrm{NP}}$ & -0.49 & {$[-0.59,-0.40]$} & 5.4 & $55 \%$ \\
$\mathscr{C}_{9}^{\mathrm{NP}}=-\mathscr{C}_{9^{\prime}}^{\mathrm{NP}}$ & -1.02 & {$[-1.18,-0.85]$} & 5.7 & $61 \%$
\end{tabular}

Figure 3: Some NP scenarios favoured in the global fit to $b \rightarrow s \ell \ell$ observables [14].

described in Sec. $2.1[13,14]$. Starting from a model hypothesis with free parameters for some Wilson coefficients $\left\{\mathscr{C}_{i}^{\mathrm{NP}}\right\}$, we perform a frequentist fit, including experimental and theoretical correlation matrices. For each one-parameter scenarios, we can give the SM-pull for each scenario, i.e. by how many sigmas the best fit point is preferred over the SM point $\left\{\mathscr{C}_{i}^{\mathrm{NP}}\right\}=0$ in the given scenario: a scenario with a large SM-pull leads to a big improvement over the SM and a better description of the data. The $p$-value is also an interesting piece of information, indicating the quality of the fit and thus the ability for the scenario to fit the experimental data (for the SM, it amounts to 9\%). The successful scenarios needs a large negative $\mathscr{C}_{9 \mu}^{\mathrm{NP}}$ [14], as can be seen from Figs. 3 and 4, with similar results when analysing subsets of data. A full 6-parameter fit of $\mathscr{C}_{7,9,10}^{\left({ }^{\prime}\right) \mathrm{NP}}$ results in a SM-pull of $5.3 \sigma$ and a $p$-value of $82 \%$, with $\mathscr{C}_{9 \mu}\left(\mathscr{C}_{10 \mu}\right)$ deviating significantly (mildly) from its SM value [13]. These results illustrate that the deviations observed in $b \rightarrow s \mu \mu$ channels follow a consistent pattern, the various deviations adding up to favour simple NP scenarios corresponding to deviations only in a few Wilson coefficients.

These results agree well with our previous analyses $[11,13,14]$ and with other recent global analyses involving similar sets of up-to-date data [15, 16]. Indeed, all analyses prefer scenarios involving a contribution to $\mathscr{C}_{9 \mu}$ in $b \rightarrow s \mu \mu$, either alone, with a contribution to $\mathscr{C}_{10 \mu}$ compatible with a $V-A$ structure or with a contribution to $\mathscr{C}_{9^{\prime} \mu}$ corresponding to right-handed currents (explaining in particular a value of $R_{K}$ close to 1 . The other Wilson coefficients are only loosely bound and compatible with SM. The hierarchy among the various NP scenarios (based on their SM-pulls) depends on specific details of the analyses and inputs, but scenarios involving contributions to $\mathscr{C}_{10 \mu}$ and/or $\mathscr{C}_{9^{\prime} \mu}$ are preferred in all analyses. These works also considered hadronic uncertainties (power corrections, form factors, charm-loop contributions): very large contributions would be needed to reproduce the anomalies in $b \rightarrow$ sll data, with difficulties to account for the consistency of deviations observed in the various channels and observables.

One may notice that Ref. [15] favours the scenario $\mathscr{C}_{9 \mu}^{\mathrm{NP}}=-\mathscr{C}_{10 \mu}^{\mathrm{NP}}$ very significantly compared to the other scenarios discussed above. This strong preference for one scenarios is supported by the other global fits which rank all scenarios more or less at the same level. This was explained in Ref. [15] by the combination of several inputs differing from the other studies: $\operatorname{Br}\left(B_{s} \rightarrow \mu \mu\right)$ (but the impact is very marginal in itself, as shown in Refs. [14, 16]), $\Lambda_{b} \rightarrow \Lambda \mu \mu$ (but once again the impact is only mild as confirmed in Ref. [17]), and $\Delta m_{s}$ (which helps sharpening their constraint on $f_{B_{s}}$ and thus increases the impact of $\operatorname{Br}\left(B_{s} \rightarrow \mu \mu\right)$ to the fit). This relies on the assumption made in Ref. [15] that there is no New Physics contribution to $\Delta F=2$ transitions in $B_{s}$ mixing and that the SM expressions for $\Delta m_{s}$ can be used relying on determinations of the SM bag parameter from LQCD simulations with very small uncertainties. It means that the results given in Ref. [15] should 

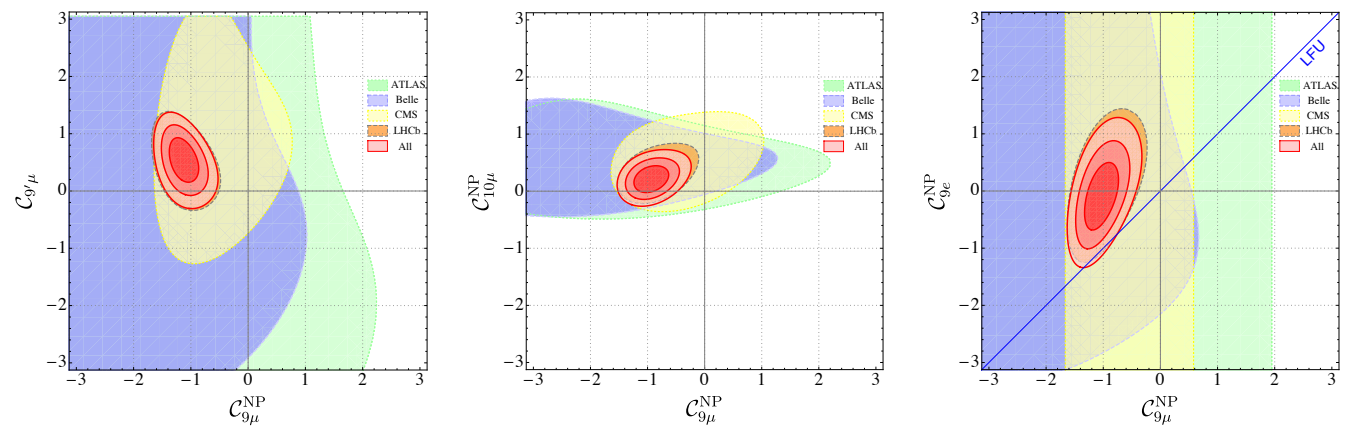

Figure 4: Results for the two-dimensional scenario with real contributions to $\mathscr{C}_{9 \mu}$ and $\mathscr{C}_{10 \mu}$ (left), $\mathscr{C}_{9 \mu}$ and $\mathscr{C}_{9^{\prime} \mu}$ (center), $\mathscr{C}_{9 \mu}$ and $\mathscr{C}_{9 e}$ (right) [14].

not be used and interpreted in the large class of NP models contributing both to $b \rightarrow \mathrm{sl \ell}$ and to $B_{s}$ mixing significantly. Other global fit analyses $[14,16]$ do not include $\Delta F=2$ observables and are thus free from this strong assumption.

In the fits, NP is allowed in $b \rightarrow$ see but not mandatory as for $b \rightarrow s \mu \mu$. It proves interesting to recast this question in terms of two kinds of NP, Lepton Flavour Universal (LFU) and Lepton Flavour Universality Violating (LFUV), so that the Wilson coefficients read [13]: $\mathscr{C}_{i e}^{\mathrm{NP}}=\mathscr{C}_{i}^{\mathrm{U}}$ and $\mathscr{C}_{i \mu}^{\mathrm{NP}}=\mathscr{C}_{i}^{\mathrm{U}}+\mathscr{C}_{i}^{\mathrm{V}}$ (with $i=9,10$ ) for $b \rightarrow s e^{+} e^{-}$and $b \rightarrow s \mu^{+} \mu^{-}$transitions respectively. Several NP scenarios fit very well the data, e.g. $\mathscr{C}_{9}^{\mathrm{U}}$ and $\mathscr{C}_{9}^{\mathrm{V}}=-\mathscr{C}_{10}^{\mathrm{V}}$ (SM-pull at $5.9 \sigma$ and $p$-value of $74 \%$ ) with vanishing contributions excluded at more than $2 \sigma$ for both LFU an LFUV NP.

Such LFU contributions arise naturally from radiative corrections. For instance the scenario that we have just discussed arises naturally when one tries to explain not only $b \rightarrow s \ell \ell$ anomalies but also the violation of LFU observed in the ratios $R_{D\left({ }^{*}\right)}$ comparing the branching ratios $B \rightarrow D\left(^{*}\right) \tau v$ and $B \rightarrow D\left(^{*}\right) \ell v(\ell=e, \mu)$ within the Standard Model Effective Field Theory [14] . The SMEFT is the effective theory obtained at the electroweak scale assuming that NP occurs at a sufficiently high scale and appears thus as dimension-6 operators to the SM Lagrangian. An operator involving $\tau$ leptons explains $R_{D^{(*)}}$ (a similar one involving muons gives a LFUV effect in $b \rightarrow s \mu^{+} \mu^{-}$). $S U(2)_{L}$ invariance leads generally to the enhancement of $b \rightarrow s \tau^{+} \tau^{-}$processes [18], and radiative corrections generate a contribution to $\mathscr{C}_{9}^{\mathrm{U}}$ [19]. This yields a correlation between $\mathscr{C}_{9}^{\mathrm{U}}$ and $R_{D^{(*)}}$ shown on the left panel of Fig. 5 (see also Ref. [15]). This scenario has a pull of $7.0 \sigma$ due to the inclusion of $R_{D^{(*)}}$.

\subsection{NP interpretations}

Since hints of LFUV are only seen in $b$-quark transitions involving leptons, it is tempting to build models explaining both $b \rightarrow c \ell v$ and $b \rightarrow s \ell \ell$ deviations simultaneously. A framework to correlate the deviations in the tree- and loop-level $b$-quark transitions is provided by the SMEFT. In this theory, two types of higher-dimension operators (scalar and tensor) are involved in the explanation of the anomalies. Under fairly general assumptions concerning the flavour structure of these operators, one can parametrise the contributions according to two couplings $C_{S}$ and $C_{T}$ that

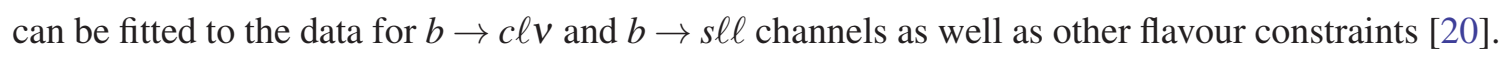



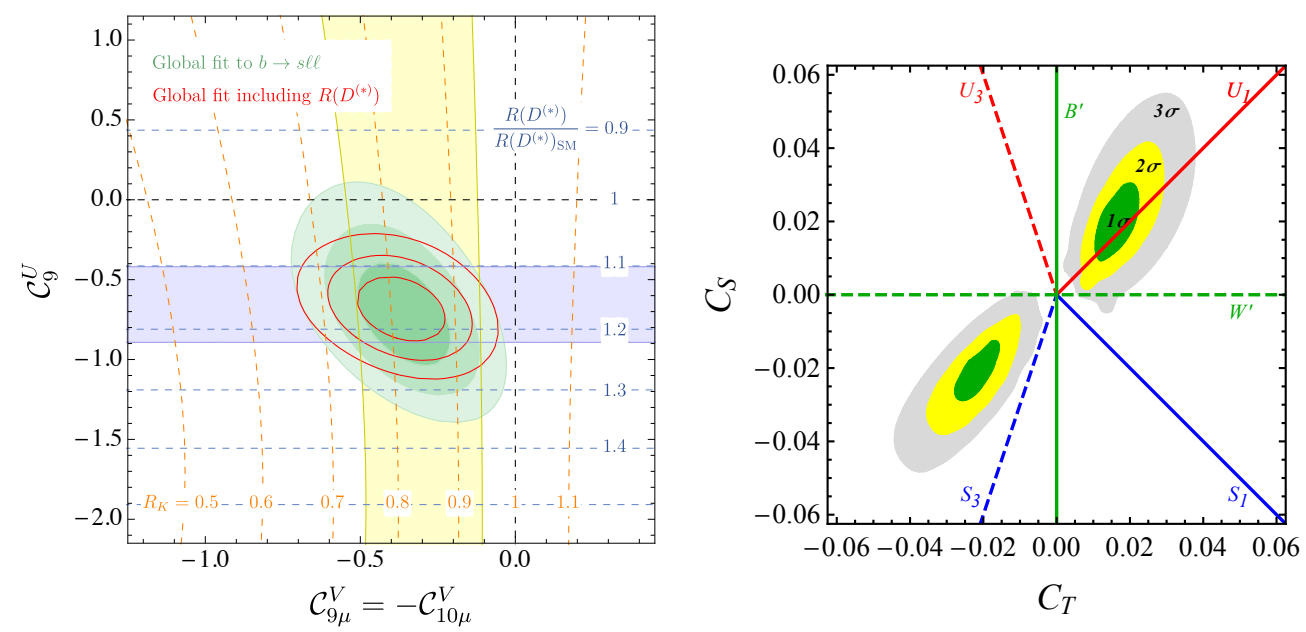

Figure 5: Left: constraints of the scenario with LFU contribution $\mathscr{C}_{9}^{\mathrm{U}}$ and LFUV contribution $\mathscr{C}_{9}^{\mathrm{V}}=-\mathscr{C}_{10}^{\mathrm{V}}$ in an EFT framework connecting $b \rightarrow c \ell v$ and $b \rightarrow s \ell v$ [14]. Right: interpretation in terms of single-mediator models for the data on $b \rightarrow c \ell v$ and $b \rightarrow s \ell \ell$, where the coloured regions correspond to a fit to flavour data and the lines to single-mediator models [20].

The result is shown on the right panel of Fig. 5 together with lines corresponding to simplified models involving a single mediator. Colourless vectors do not seem to be able to accommodate all the data $\left(B_{s} \bar{B}_{s}\right.$ mixing, direct production at the LHC). Scalar leptoquarks may in principle provide a good fit to the data, but the radiative corrections to $Z \rightarrow t \bar{t}$ and $Z \rightarrow v v$ are significant and create a mild tension with $R_{D^{*}}$. In both cases, these problems can be solved by including further fields in the theory. Finally, the vector leptoquark singlet $U_{1}$ fit very well the data. Such a massive vector leptoquark requires additional fields to build an ultraviolet complete model, for which several possibilities have been investigated further, such as Pati-Salam models or partial compositeness. These models feature additional particles, such as heavy vector-like quarks and leptoquarks [1].

\section{New observables}

\subsection{Relevance of angular observables}

We see that several NP scenarios with a similar ability to explain the deviations observed in $b \rightarrow$ sll data (SM pull, $p$-value) in a very economical way, showing the consistency of these patterns of deviations. A better theoretical understanding in the hadronic uncertainties (in particular charm loops) would help to disentangle these scenarios, as well as more precise measurements. But an even more powerful approach would consist in new observables that would provide further constraints on NP contributions, with different theoretical and experimental uncertainties attached to their interpretations. We will only discuss here modes that are allowed in the Standard Model: searches for lepton-flavour violating modes provide interesting constraints for NP models, but they cannot enter the global fit analyses outlined above in a model-independent way, since they correspond to operators associated with a different kind of effective Hamiltonian from the one considered up to now. 
It is possible to formulate the helicity amplitude analysis of $b \rightarrow s \ell \ell$ transitions for a $b$-hadron decaying into two hadrons through an intermediate resonance of given spin in a very general way [21]. In the resulting angular distribution, each angular coefficient corresponds to interference terms between pairs of helicity amplitudes, each of which is a linear combination of Wilson coefficients and form factors (non-local $c \bar{c}$ contributions are taken into account by adding a longdistance hadron- and kinematics-dependent piece to the Wilson coefficients). The senstivity to short-distance NP contributions depends therefore on the hadronic physics involved (form factors...), but also on purely kinematic factors (masses of the initial and final hadrons, kinematic range allowed for the lepton pair). For instance $B \rightarrow K^{*} \mu \mu$ exhibits a sensitivity to $\mathscr{C}_{7}-\mathscr{C}_{7}$ and $\mathscr{C}_{9 \mu}-\mathscr{C}_{9^{\prime} \mu}$, whereas $B \rightarrow K \mu \mu$ is sensitive to $\mathscr{C}_{9 \mu}+\mathscr{C}_{9^{\prime} \mu}$ and (marginally) $\mathscr{C}_{7}+\mathscr{C}_{7^{\prime}}$.

The kinematic range allowed drives the methods to be used to tackle the hadronic dynamics, at the level of the form factors (light-cone sum rules, LQCD simulations) and the amplitudes (softcollinear effective theory and QCD factorisation at large recoil of the final hadron, heavy-quark effective theory and operator product expansion at low recoil). It proves often easier to deal with charm-loop contributions at low recoil, using estimates of the amount of quark-hadron duality violation [22], whereas the large recoil region would require a more involved analysis following the light-cone sum rule approach of Ref. [3] supplemented with experimental inputs similarly to Ref. [9].

\subsection{New observables related to modes already studied}

A first avenue consists in exploiting modes that have already been measured in detail in order to extract further observables. For instance, CP-violating asymmetries have been measured for $B \rightarrow K^{*} \mu \mu$ and $B_{s} \rightarrow \phi \mu \mu$, which helps in constraining the imaginary part of the NP contribution to Wilson coefficients [1]. It has also been proposed to perform the time-dependent analysis of $B_{d} \rightarrow K^{*}\left(\rightarrow K_{S} \pi^{0}\right) \mu \mu$ and $B_{s} \rightarrow \phi(\rightarrow K \bar{K}) \mu \mu$ in order to define new observables resulting from the interference between the neutral-meson mixing and the helicity amplitudes describing the $b \rightarrow$ sll decay, leading to observables with some sensitivity to right-handed currents [23].

One can also think of defining angular observables that are sensitive to the violation of LFU, by comparing the angular distribution of $B \rightarrow K^{*} \mu \mu$ and $B \rightarrow K^{*} e e$ (for instance). Disentangling the NP scenarios currently allowed can be achieved by considering differences of optimised observables like $Q_{5}=P_{5 \mu}^{\prime}-P_{5 e}^{\prime}[7,24]$ rather than differences of the angular coefficients obtained directly from an angular analysis [25]. The Belle experiment has measured some of these coefficients [1], hinting at a deviation in agreement with the NP scenarios favoured by global fits, but with too large uncertainties to draw any firm conclusion yet.

Even though they are particularly challenging from the experimental point of view [26], $b \rightarrow$ $s \tau \tau$ modes could also be particularly interesting to disentangle NP scenarios. First they would provide a further test of LFU, and one could expect that they are even more sensitive to the violation of LFU than muons. Moreover, explanations at the level of effective field theories of the deviations in $b \rightarrow c \ell v$ and $b \rightarrow s \ell \ell$ may generate huge enhancements of the $b \rightarrow s \tau \tau$ modes, making them more accessible to current and forthcoming experiments (Belle II, LHCb, CMS...) [18]. If they can be observed, these modes may provide interesting tests of terms suppressed by $m_{\ell}^{2} / q^{2}$ in the angular distributions, in particular the scalar and pseudoscalar contributions. The subsequent decay 
of the $\tau$ lepton can provide interesting information on the polarisation of the $\tau$ [27], leading to a more detailed understanding of the contributions of the various helicity amplitudes to the decay.

\subsection{New observables related to new modes}

A second avenue consists in exploiting modes that have not been observed (or only partially). In the case of mesons, one can think of analysing $B \rightarrow K \pi \mu \mu$ at higher $K \pi$ invariant mass, where several excited $K^{*}$ resonances of spin from 0 to 2 (around 1410-1430 MeV) and up to 4 (around $2100 \mathrm{MeV}$ ) are accessible. The LHCb collaboration have performed an angular analysis in the former window [28]. Form factors and charm-loop effects have not been extensively investigated for these resonances, which limits currently their exploitation in the contex of global fits. However, some observables for $B \rightarrow K_{J} \mu \mu$ with $J \leq 4$ have been studied in Refs. [29]. In the case $J=2$ [30], the angular structure is very similar to $B \rightarrow K^{*} \mu \mu$ in the absence of tensor operators (as discussed in Ref. [21]), so that optimised observables similar to those in $B \rightarrow K^{*} \mu \mu$ can be defined. It remains to be seen if the separation of the different resonances in the range $1410-1430 \mathrm{MeV}$ can be performed experimentally and if hadronic effects can be computed accurately for wider resonances of this kind.

Rare $\Lambda_{b}$ decays offer completely different theoretical and experimental environments, with further possibilities offered by the spin of the initial baryon and the possibility for the light baryon resulting from the $b \rightarrow$ sll transition to decay weakly. The decay $\Lambda_{b} \rightarrow \Lambda(\rightarrow p \pi) \mu \mu$ has been studied in many references [31], showing deviations from the SM in the branching ratio and some of the angular observables [32], but with rather large uncertainties, so that the model-independent analysis of these results agrees both with the SM and with NP interpretations already hinted at in rare meson decays [17, 33].

Another promising possibility consists in looking at decays of the $\Lambda_{b}$ baryon into excited $\Lambda$ states through the decay chain $\Lambda_{b} \rightarrow \Lambda^{*}(\rightarrow p K) \ell \ell$, which is expected to be dominated by the narrow $\Lambda(1520)$ that appears prominently in the pentarquark searches using $\Lambda_{b} \rightarrow p K J / \psi$ [34]. A first theoretical study of the decay $\Lambda_{b} \rightarrow \Lambda(1520) \ell \ell$ was proposed in Ref. [35], where the angular distribution was derived and the sensitivity of the angular observables on hadronic uncertainties and NP contributions was investigated. The branching ratio for $\Lambda_{b} \rightarrow \Lambda(1520) \mu \mu$ was shown to decrease for the NP scenarios favoured by the global fits. Recently, the LHCb experiment has measured the ratio $R_{p k}$ comparing $\operatorname{Br}\left(\Lambda_{b} \rightarrow p K \mu \mu\right)$ and $\operatorname{Br}\left(\Lambda_{b} \rightarrow p K e e\right)$. The result is compatible with SM expectations, but the central value suggests a suppression of $\operatorname{Br}\left(\Lambda_{b} \rightarrow p K \mu \mu\right)$ compared $\operatorname{Br}\left(\Lambda_{b} \rightarrow p K e e\right)$, which agrees with the results obtained in Ref. [35] if one assumes that $R_{p k}$ is dominated by the contribution of $\Lambda(1520)$. It would thus be particularly interesting to identify in $R_{p K}$ the fraction of events due to the $\Lambda(1520)$ excited state, and to measure the angular distribution of the decay through this intermediate excited state.

\section{Outlook}

$b$-quark decays exhibit interesting patterns of deviations with respect to SM predictions in two different sectors: the tree-level charged-current transitions $b \rightarrow c \ell v_{\ell}$ and the loop-level neutralcurrent processes $b \rightarrow s \ell \ell$. In the first case, a few observables are available (branching ratios) whereas in the latter case, many quantities (branching ratios, angular observables) are measured. 
Interestingly, both channels suggest NP contributions distinguishing between the various leptons. Fits to additional NP couplings within the effective Hamiltonian approach can be performed to understand the pattern of deviations exhibited in the various $b \rightarrow s \ell \ell$ modes already measured. Hadronic uncertainties play a significant role here and should be assessed more precisely in the future. Talking into account these uncertainties, the global fit analyses are still able to identify economical scenarios explaining the observed patterns of deviations consistently by shifts in a few short-distance Wilson coefficients. Various NP models have been proposed to explain these anomalies (leptoquarks, partial compositeness, extended electroweak gauge sector...). In order to discriminate among these models, it is clear that more data from LHCb and Belle-II is needed and that additional observables and modes should be studied to confirm or disprove these anomalies, and several classes of such observables and modes have already been identified and discussed. Thanks to the potential of $b \rightarrow s \ell \ell$ transitions to open windows of the physics beyond the Standard Model, it is clear that this subject will remain a topic of intense discussion both experimentally and theoretically in the coming years.

\section{Acknowledgments}

This project has received funding from the European Unions Horizon 2020 research and innovation programme under the Marie Sklodowska-Curie grant agreements No 690575 and 674896.

\section{References}

[1] S. Bifani, S. Descotes-Genon, A. Romero Vidal and M. H. Schune, J. Phys. G 46 (2019) 023001 [arXiv: 1809.06229 [hep-ex]].

[2] S. Descotes-Genon et al., JHEP 1301 (2013) 048 [arXiv:1207.2753 [hep-ph]] and JHEP 1305 (2013) 137. [arXiv:1303.5794 [hep-ph]].

[3] A. Khodjamirian et al., JHEP 1009 (2010) 089. [arXiv:1006.4945 [hep-ph]].

[4] A. Bharucha, D. M. Straub and R. Zwicky, JHEP 1608 (2016) 098. [arXiv:1503.05534 [hep-ph]]. N. Gubernari, A. Kokulu and D. van Dyk, JHEP 1901 (2019) 150 [arXiv:1811.00983 [hep-ph]].

[5] S. Descotes-Genon, A. Khodjamirian and J. Virto, JHEP 1912 (2019) 083 [arXiv:1908.02267 [hep-ph]].

[6] F. Kruger and J. Matias, Phys. Rev. D 71 (2005) 094009 [hep-ph/0502060].

J. Matias et al., JHEP 1204 (2012) 104 [arXiv:1202.4266 [hep-ph]].

C. Bobeth, G. Hiller and D. van Dyk, JHEP 1007 (2010) 098 [arXiv:1006.5013 [hep-ph]].

[7] B. Capdevila et al., JHEP 1610 (2016) 075 [arXiv:1605.03156 [hep-ph]].

[8] T. Blake et al., Eur. Phys. J. C 78 (2018) 453 [arXiv:1709.03921 [hep-ph]].

[9] C. Bobeth et al., Eur. Phys. J. C 78 (2018) 451 [arXiv:1707.07305 [hep-ph]].

[10] A. Ali, G. F. Giudice and T. Mannel, Z. Phys. C 67 (1995) 417 [hep-ph/9408213].

G. Hiller and F. Kruger, Phys. Rev. D 69 (2004) 074020 [hep-ph/0310219].

S. Descotes-Genon et al., JHEP 1106 (2011) 099 [arXiv:1104.3342 [hep-ph]].

W. Altmannshofer, P. Paradisi and D. M. Straub, JHEP 1204 (2012) 008 [arXiv:1111.1257 [hep-ph]].

C. Bobeth, G. Hiller and D. van Dyk, Phys. Rev. D 87 (2013) 034016 [arXiv:1212.2321 [hep-ph]]. 
[11] S. Descotes-Genon, J. Matias and J. Virto, Phys. Rev. D 88 (2013) 074002. [arXiv:1307.5683 [hep-ph]].

[12] W. Altmannshofer and D. M. Straub, Eur. Phys. J. C 73 (2013) 2646 [arXiv:1308.1501 [hep-ph]]. F. Beaujean, C. Bobeth and D. van Dyk, Eur. Phys. J. C 74 (2014) 2897 [Err: Eur. Phys. J. C 74 (2014) 3179] [arXiv:1310.2478 [hep-ph]].

[13] S. Descotes-Genon et al, JHEP 1606 (2016) 092 [arXiv:1510.04239 [hep-ph]].

B. Capdevila et al., JHEP 1801 (2018) 093 [arXiv:1704.05340 [hep-ph]].

M. Algueró et al., Phys. Rev. D 99 (2019) 075017 [arXiv:1809.08447 [hep-ph]].

[14] M. Algueró et al., Eur. Phys. J. C 79 (2019) 714 [arXiv:1903.09578 [hep-ph]].

[15] J. Aebischer et al., arXiv:1903.10434 [hep-ph];

[16] A. K. Alok et al., JHEP 1906 (2019) 089 [arXiv:1903.09617 [hep-ph]]. M. Ciuchini et al., Eur. Phys. J. C 79 (2019) 719 [arXiv:1903.09632 [hep-ph]].

K. Kowalska, D. Kumar and E. M. Sessolo, Eur. Phys. J. C 79 (2019) 840 [arXiv:1903.10932 [hep-ph]].

A. Arbey et al., Phys. Rev. D 100 (2019) 015045 [arXiv:1904.08399 [hep-ph]].

G. D’Amico et al., JHEP 1709 (2017) 010 [arXiv:1704.05438 [hep-ph]].

[17] T. Blake, S. Meinel and D. van Dyk, arXiv:1912.05811 [hep-ph].

[18] B. Capdevila et al., Phys. Rev. Lett. 120 (2018) 181802 [arXiv:1712.01919 [hep-ph]].

[19] A. Crivellin et al., Phys. Rev. Lett. 122 (2019) 011805 [arXiv:1807.02068 [hep-ph]].

[20] D. Buttazzo et al., JHEP 1711 (2017) 044 [arXiv:1706.07808 [hep-ph]].

[21] J. Gratrex, M. Hopfer and R. Zwicky, Phys. Rev. D 93 (2016) 054008 [arXiv:1506.03970 [hep-ph]]. B. Dey, Phys. Rev. D 95 (2017) 033004 [arXiv:1609.06115 [hep-ph]].

[22] M. Beylich, G. Buchalla and T. Feldmann, Eur. Phys. J. C 71 (2011) 1635 [arXiv:1101.5118 [hep-ph]].

[23] S. Descotes-Genon and J. Virto, JHEP 1504 (2015) 045 Erratum: [JHEP 1507 (2015) 049] [arXiv:1502.05509 [hep-ph]].

[24] M. Algueró et al., JHEP 1907 (2019) 096 [arXiv:1902.04900 [hep-ph]].

[25] W. Altmannshofer, P. Stangl and D. M. Straub, Phys. Rev. D 96 (2017) 055008 [arXiv:1704.05435 [hep-ph]].

[26] R. Aaij et al. [LHCb Collaboration], Phys. Rev. Lett. 118 (2017) 251802 [arXiv:1703.02508 [hep-ex]].

J. F. Kamenik et al., Eur. Phys. J. C 77 (2017) 701 [arXiv:1705.11106 [hep-ph]].

[27] K. Hagiwara, A. D. Martin and D. Zeppenfeld, Phys. Lett. B 235 (1990) 198.

[28] R. Aaij et al. [LHCb Collaboration], JHEP 1612 (2016) 065 [arXiv:1609.04736 [hep-ex]].

[29] C. D. Lu and W. Wang, Phys. Rev. D 85 (2012) 034014 [arXiv:1111.1513 [hep-ph]].

B. Dey, Phys. Rev. D 95 (2017) 033004 [arXiv:1609.06115 [hep-ph]].

[30] D. Das et al., Phys. Rev. D 99 (2019) 093012 [arXiv:1812.11803 [hep-ph]]. 
[31] T. Gutsche et al., Phys. Rev. D 87 (2013) 074031 [arXiv:1301.3737 [hep-ph]].

P. Böer, T. Feldmann and D. van Dyk, JHEP 1501 (2015) 155 [arXiv:1410.2115 [hep-ph]].

S. Roy, R. Sain and R. Sinha, Phys. Rev. D 96 (2017) 116005 [arXiv:1710.01335 [hep-ph]].

D. Das, JHEP 1807 (2018) 063 [arXiv:1804.08527 [hep-ph]].

D. Das, Eur. Phys. J. C 78 (2018) 230 [arXiv:1802.09404 [hep-ph]].

T. Blake and M. Kreps, JHEP 1711 (2017) 138 [arXiv:1710.00746 [hep-ph]].

[32] R. Aaij et al. [LHCb Collaboration], JHEP 1506 (2015) 115 Erratum: [JHEP 1809 (2018) 145] [arXiv:1503.07138 [hep-ex]] and JHEP 1809 (2018) 146 [arXiv:1808.00264 [hep-ex]].

[33] W. Detmold and S. Meinel, Phys. Rev. D 93 (2016) 074501 [arXiv:1602.01399 [hep-lat]].

[34] R. Aaij et al. [LHCb Collaboration], Phys. Rev. Lett. 115 (2015) 072001 [arXiv:1507.03414 [hep-ex]].

[35] S. Descotes-Genon and M. Novoa Brunet, JHEP 1906 (2019) 136 [arXiv:1903.00448 [hep-ph]]. 